\title{
Gastroenteritis eosinofílica: a propósito de dos casos
}

\author{
J. F. ÁLVAREZ GARCÍA, M. T. SÁNCHEZ SÁNCHEZ, M. CHIQUERO PALOMO, A. \\ COSTO CAMPOAMOR, J. M. SAPONI CORTÉS, F. PÉREZ REYES, J. LUENGO \\ ÁLVAREZ
}

Servicio de Medicina Interna. Complejo Hospitalario de Cáceres. Hospital San Pedro de Alcántara. Cáceres

\author{
EOSINOPHILIC GASTROENTERITIS: TWO CASES REPORT
}

\begin{abstract}
RESUMEN
La gastroenteritis eosinofílica es una enfermedad poco frecuente, caracterizada por la presencia de un infiltrado eosinófilo que puede afectar a las distintas capas de la pared intestinal. Las manifestaciones clínicas son variables y los síntomas dependen del grado de infiltración y del número de capas afectadas. Se caracteriza por presentar, en la mayoría de los casos, eosinofília periférica. Su diagnóstico definitivo es anatomopatologico. Aportamos dos casos, ambos con excelente respuesta al tratamiento esteroideo.
\end{abstract}

PALABRAS CLAVE: Gastroenteritis eosinofílica. Eosinofilia. Tratamiento esteroideo. Ascitis eosinofílica.

\begin{abstract}
Eosinophilic gastroenteritis is a rare condition characterized by eosinophilic infiltration or the different layers of the intestinal wall. Clinical symptoms depend of the site of eosinophilic infiltration and the layers involved. Usually characterized by peripheral eosinophilia. Definitive diagnosis is based on histopathological findings. We report two cases with excellent response to steroid therapy.
\end{abstract}

KEY WORDS: Eosinophilic gastroenteritis. Eosinophilia. Steroid therapy. Eosinophilic ascites.

Álvarez García JF, Sánchez Sánchez MT, Chiquero Palomo M, Costo Campoamor A, Saponi Cortés JM, Pérez, Reyes F, Luengo Álvarez J. Gastroenteritis eosinofílica: a propósito de dos casos. An Med Interna (Madrid) 2004; 21: 447-449.

\section{INTRODUCCIÓN}

La gastroenteritis eosinofílica (GEE) es una entidad infrecuente, descrita por primera vez por Kaijser en 1937 (1), con menos de 300 casos publicados en todo el mundo (2), de los que 51 corresponden a nuestro pais (MEDLINE 1966-2003). $\mathrm{Su}$ etiopatogenia es desconocida. Se caracteriza por la presencia de un infiltrado eosinofílico en la pared del tubo digestivo. La clínica y evolución de la enfermedad es variable en función de la localización del área del tracto digestivo afecta y del grado de infiltración de la pared intestinal. Presentamos dos nuevos casos, con diferentes formas de presentación clínica, con excelente respuesta a los esteroides, permaneciendo asintomáticos actualmente sin necesidad de tratamiento.

\section{CASOS APORTADOS}

Caso 1: Varón de 60 años, exfumador de 15 cig./día desde hace dos años, remitido a consulta por cuadro de un mes de evolución de astenia, hiporexia, pérdida de $2-3 \mathrm{~kg}$ de peso junto con dolor abdominal episódico, localizado en epigastrio y sin relación con la ingesta. Refería clínica compatible con episodio autolimitado de hemorragia digestiva alta, pero que no acudió a su médico para ser valorada. Había recibido tratamiento con antisecretores sin experimentar mejoría. No refería fiebre ni alteraciones del ritmo intestinal. En la exploración física únicamente destacaba dolor selectivo a la palpación profunda en epigastrio, sin signos de irritación peritoneal. Pruebas complementarias: hemograma 6.000 leucoc. (95\% eosinófilos), resto de hemograma y VSG normales. Bioquímica, coagulación y proteinograma normales. Inmunoglobulinas (incluida Ig E) normales. ANA, ENAs, ANCA, Anticuerpos anti-células apriétales, anti-factor intrínseco, antireticulina, antiendomisio, antitransglutaminasa y antigliadina normales o negativos. Examen de huevos y parásitos en heces negativo. RX de tórax y abdomen normales. Tránsito abdominal normal. Ecografía abd.: imágenes compatibles con quistes simples a nivel hepático, resto de la exploración normal. Gastroscopia: mucosa antral con aspecto atrófico y nodular. Biopsia gástrica: gastritis crónica atrófica con metaplasia intestinal inactiva con importante infiltración eosinofílica. Biopsia duodenal: signos inflematorios inespecíficos con intensa infiltración por eosinófilos. Se inició trata-

Trabajo aceptado: 1 de abril de 2004

Correspondencia: Alberto Costo Campoamor. C/ Isla de Hierro, 6, 5 Y. 10001 Cáceres. e-mail: a.costo@ terra.es 
miento con prednisona a dosis de $40 \mathrm{mg} /$ día con desaparición de la sintomatología digestiva en 4-5 días. Los esteroides se retiraron paulatinamente, manteniéndose asintomático y con eosinofilia periférica controlada actualmente.

Caso 2: Varón de 27 años, fumador de 10 cig/día, amigdalectomizado y adenodectomizado en la infancia. Ingresa por cuadro de 6 semanas de evolución de astenia, pérdida ponderal de unos $10 \mathrm{~kg} \mathrm{y}$ dolor abdominal en piso superior de tipo cólico recurrente (había acudido en varias ocasiones a Urgencias, recibiendo medicación gastroprotectora y procinética sin notar mejoría). Presentó episodio único de deposiciones diarreicas sin productos patológicos al inicio del cuadro. La exploración física reveló un abdomen distendido con semiología ascítica positiva sin signos de irritación peritoneal. Examenes complementarios: hemograma 15.700 leucoc (35\% eosinófilos), con serie roja y plaquetaria normales. La bioquímica general, coagulación y VSG eran normales. Las determinaciones complementarias de proteinograma, inmunoglobulinas (incluida Ig E), marcadores tumorales digestivos, PCR, ANA, ENAs y ANCA fueron normales o negativos. La RX de abdomen mostró un velamiento difuso. Se realizó paracentesis diagnóstica con salida de líquido de aspecto turbio que cumplía criterios bioquímicos de exudado, con cultivos negativos y celularidad inflamatoria con predominio de eosinófilos. TAC toraco-abdominal: ascitis moderada, discreta dilatación de asas yeyunales e ileales y pequeñas adenopatías en al raiz del mesenterio con discreto engrosamiento del íleon terminal. Gastroscopia: gastritis eritematosa con pliegue duodenal engrosado. Biopsia duodenal: vellosidades intestinales conservadas con marcada infiltración difusa por eosinófilos que afectaba la pared intestinal en todo su espesor. Se instauró tratamiento con prednisona a dosis de $40 \mathrm{mg} / \mathrm{día}$ con remisión de los síntomas en una semana, manteniéndose asintomático actualmente.

\section{DISCUSIÓN}

La gastroenteritis eosinofílica (GEE) es una entidad clínico-patológica de presentación rara, con una mayor descripción de casos en los últimos diez años, probablemente en relación con un mayor índice de sospecha de esta enfermedad y una mayor disponibilidad de técnicas diagnósticas invasivas como la biopsia endoscópica digestiva $(3,4)$. Puede presentarse en cualquier edad, aunque es más frecuente entre la tercera y quinta décadas de la vida, siendo excepcional antes de los 20 años $(3,6,7)$. La etiopatogenia de la GEE no está totalmente aclarada: la presencia de eosinofilia tisular y sanguínea, la mayor incidencia de trastornos alérgicos (rinitis, urticaria, asma bronquial, etc), los niveles elevados de Ig E y la respuesta a los esteroides apoyan la hipóteis de una reacción de hipersensibilidad tipo I a ciertos alimentos (presente en el 50\% de los casos) $(3,5,8)$; y otras veces mediados por Ig E $(9,10)$.

Cursa con diferentes manifestaciones clínicas según su localización en el tracto digestivo, pudiendo afectar a cualquier tramo desde el esófago (4) hasta el colon (11), y la capa de la pared en la que predomine el infiltrado eosinófilo (aunque a menudo las características clínicas se superponen porque pueden estar afectadas varias capas (5) ). Se presenta con mayor frecuencia en el estómago y en el intestino delgado proximal, que por otra parte, son los tramos más frecuentemente biopsiados $(3,5)$. Klein y cols. (7) describieron tres formas de presentación clínica según la capa de la pared intestinal predominantemente afecta. La afectación de la mucosa cursa con dolor abdominal, nauseas, vómitos, diarrea y pérdida de peso, asociándose en las formas graves con un síndrome malabsortivo. Es la forma más frecuente y en la que se ha visto una mayor relación de antecedentes alérgicos (hasta en el
$50 \%$ de los pacientes) (5). La forma con afectación de la capa muscular se caracteriza por un cuadro de oclusión o suboclusión intestinal secundario a un engrosamiento y rigidez de la pared intestinal, preferentemente a nivel gástrico y la primera porción del intestino delgado, siendo responsable de la presentación en forma de abdomen agudo (12-14). La forma clínica más rara es la afectación de la capa serosa, que se asocia al desarrollo de ascitis eosinofílica por inflamación local del peritoneo adyacente (como en el segundo de los casos aportados). Manifestaciones menos comunes son la disfagia (4), hemorragia digestiva (15), visceromegalias y adenopatías periféricas $(16)$ o derrame pleural $(3,12)$. El dato analítico más frecuente es la eosinofilia en sangre periférica aunque no tiene valor como criterio diagnóstico al estar ausente en un porcentaje variable de casos $(20-80 \%)(2,5,17)$. Es característica la presencia de un VSG normal o ligeramente elevada $(<20 \mathrm{~mm}$ a la primera hora ). El resto de parámetros analíticos suelen ser normales, excepto cuando hay un síndrome de malabsorción, en cuyo caso se presentan las alteraciones propias del mismo ( anemia ferropénica, hipoproteinemia, etc.).

Los criterios que definen la GEE son la presencia de síntomas gastrointestinales, la infiltración eosinófila de una o más áreas gastrointestinales demostradas por biopsia, la ausencia de compromiso eosinofílico de otros órganos fuera del aparato digestivo, y la ausencia de infestación parasitaria (5). El estudio baritado del tracto gastrointestinal suele ser patológico, apreciándose signos como engrosamiento de pliegues y zonas estenosadas en grado variable, aunque de carácter inespecífico $(18,19)$. En el estudio endoscópico deben realizarse múltiples biopsias ya que la enfermedad puede afectar diferentes capas de la pared intestinal y tiende a producirse de forma parcheada. Por este motivo deben obtenerse muestras tanto de zonas afectas como de zonas aparentemente normales, siendo las áreas preferentes el antro gástrico y el duodeno, por ser los sitios donde es mas común que se hallen estas anormalidades anatomopatológicas (5). Está indicada su realización de forma repetida en aquellos pacientes que presenten síntomas gastrointestinales inexplicables, eosinofilia periférica y biopsias previas negativas (20). La laparoscopia constituye actualmente un procedimiento alternativo eficaz en los casos de diagnóstico difícil no accesible por otras técnicas (21).

El diágnostico diferencial de la GEE debe realizarse con cuadros de sintomatología inespecífica abdominal que aparecen asociados a infiltrado eosinofílico del tubo digestivo (2): parasitosis intestinales, enfermedad inflamatoria intestinal, síndrome hipereosinofílico primario, afectación intestinal por enfermedades reumáticas, linfomas, adenocarcinomas, mastocitosis sistémica, pólipo fibroide inflamatorio y alergias farmacológicas.

Los pacientes con síntomas escasos y esporádicos deben ser tratados de forma conservadora con actitud expectante (5), seguimiento clínico y tratamiento sintomático si es preciso. El tratamiento dietético parace útil si extisten antecedentes de hipersensibilidad alimentaria (9). Los corticoides constituyen la base del tratamiento, especialmente en las formas con afectación serosa y muscular (5). Las dosis utilizadas en adultos están entre 20-40 mg/día de prednisona, con disminución progresiva hasta su suspensión. En ocasiones, es necesario establecer un tratamiento de mantenimiento con dosis bajas de prednisona $(5-10 \mathrm{mg} /$ día $)$ por recaídas en el cuadro clínico. Algunos autores han utilizado el cromoglicato disódico en caso de ausencia de respuesta, refractariedad 
o contraindicación del tratamiento corticoideo (22-24). Tambien se ha utilizado un antihistamínico (ketotifeno) con los mismos objetivos pero peor respuesta $(25,26)$; y en ocasiones inmunosupresores (azatioprina o ciclosporina) (2). Recientemente se ha preconizado el montelukast -antagonista de los leucotrienos- con buenos resultados en el tratamiento de la gastroenteritis eosinofílica $(27,28)$. A pesar de que en muchas ocasiones la GEE se identifica, o al menos se sospecha, clínicamente, en ciertas circunstancias surgen complicaciones como obstrucción intestinal, perforación o sangrado, siendo entonces indicación de tratamiento quirúrgico (29). El pronóstico es bueno. Su evolución es imprevisible con remisiones espontáneas o inducidas por esteroides, pudiendo adoptar un curso recidivante sin secuelas a largo plazo. La mortalidad es excepcional, generalmente en relación con complicaciones quirúrgicas (3). La obstrucción es la complicación aguda más común. No hay un mayor riesgo de desarrollar un cáncer gastrointestinal (5).

\section{Bibliografía}

1. Kaijser R. Zur kentuis der allergischen affektioner desima verdanungskanal von standpunk desima chirurgen aus. Arch Klin Chir 1937; 188: 36-64.

2. Talley NJ. Eosinophilic gastroenteritis. En: Feldman M, Schardschmidt BF, Sleisenger MH, eds. Sleissenger and Fortran's Gastrointestinal and Liver Disease. 6th. de. Philadelphia, WB Saubders. 1998: 1679-1688.

3. Ruiz Montes F, Reñé JM, Rubio M. Gastroenteritis eosinofílica: Revisión de los casos publicados en España y comparación con la literatura extranjera. Rev Esp Enf Ap Digest 1992; 81: 270-279.

4. Borda F, Jiménez FJ, Martinez Peñuela JM, Echarri A, Martín Granizo y Aznarez R. Esofagitis eosinofílica: ¿ Una entidad infradiagnosticada? Rev Esp Enf Ap Digest 1996; 88: 701-704.

5. Talley NJ, Shorter RG, Phillips SF, Zinsmeister AR. Eosinophilic gastroenteritis: a clinicopathologicla study of patients with disease of the mucosa, cuscle layer and subserosal tissues. Gut 1990; 31: 54-58.

6. Lee CM, Changchien CS, Chen PC, Lin DY, Sheen IS and cols. Eosinophilic gastroenteritis: 10 years experience. Am J Gastroenterol 1993; 88: 70-74.

7. Kein NC, Hargrove RL, Sleissenger Mh, Jeffries GH. Eosinophilic gastroenteritis. Medicine (Baltimore) 1970; 49: 299-319.

8. Weller PF. The inmunobiology of eosinophilis. N Engl J Med 1991; 324: 110-115.

9. Scudamare HH, Phillips SF, Swedlund HA, Gleich GJ. Food allergy manifested by eosinophilia, elevated inmunoglobulin E level, and protein-losing enteropathy: The syndrome of allergic gastroenteropathy. J Allergy Clin Inmunol 1982; 70: 129-134

10. Caldwell JH, Tennevaum JI, Bronstein HA. Serum Ig E in eosinophilic gastroenteritis. N Engl J Med 1975; 292: 1388-1390.

11. Shweiki E, West JC, Kena JW, Kelley SE, Thompson A, Bross RJ, Tyler WB. Eosinophilic gastroenteritis presenting as an abstructing cecal mass: a case report and review of the literature. Am J Gast 1999; 94: 3644-3645.

12. Valderrama Rojas M, Ceballos Porras A, Pérez Tamayo J, Filgueira Rubio J, Rodríguez Gorostiza J, Hergueta Martín-Hartajo L. Abdomen agudo como forma de presentación de enteritis eosinofílica. An Med Interna (Madrid) 2001; 18: 450-451.

13. García-Sancho Téllez L, Hardisson Hernáez D, García-Sancho Martín L, Martínez Jiménez P. Abdomen Agudo por gastroenteritis eosinofílica: revisión de 11 casos. Cir Esp 1996; 60 (4): 274-281.

14. Perea García J, Ots Gutiérrez JR, Martín Ruiz JR, Quijada García B,

Rodríguez Martín. Gastroenteritis eosinofílica como causa de obstrucción intestinal. Cirugía Española 2001; 64 (4): 431-432.

15. Caldwell JH, Mekhijan HS, Hurtubise PE, Benam FM. Eosinophilic gastroenteritis with obstruction. Inmunologicla studies of seven patients. Gastroenterology 1978; 74: 825-828.

16. Robert F, Omura E, Durant J. Mucosal eosinophilic gastroenteritis with systemic involvement. Am J Med 1977; 62: 139-143.

17. Naylor AR. Eosinophilic gastroenteritis. Scott Med J 1990; 35: 163 165.

18. Remacha Tomey B, Palau A, Colom J, Ripollés V. Gastroenteritis eosinofílica. An Med Interna (Madrid) 1998; 15: 554-559.

19. Stalmeyer MJB, Chew FS. Eosinophilic gastroenteritis. Am J Roetgenol 1993; 161: 296.

20. De la Serna Higuera C, Rodríguez Gómez S, Martín Arribas M, Martinez Moreno J. Gastroenteritis eosinofílica: espectro clínico de una misma entidad. An Med Interna (Madrid) 2002; 19: 361-364.

21. Rodríguez HE, Djohan RS, Chill WJ. Laparoscopic diagnosis of eosinophilic gastroenteritis. JS Laparosc Surg 1998; 2: 181-184.

22. Di Gioachino X, Pizzicanella G, Frenni W. Sodium cromoglycate in the treatment of eosinophilic gastroenteritis. Allergy 1990; 45: 161-166.

23. Pérez- Millán A, Martín-Lorente JL, López-Morante A, Yuguero L, Sáez Royuela F. Subserosal eosinophilic gastroenteritis treated efficaciously with sodium cromoglycate. Dig Dis Sci 1997; 20(1): 21-23.

24. Gjersoe P, Rasmussen SN, Hansen BF. Eosinophilic enteritis. Vgeshr Laeger 2000; 162: 2756-2757.

25. Mehammed Y, Feanny SJ, Shermann PM. Benefit of ketotifen in patients with eosinophilic gastroenteritis. Am J Med 1991; 90: 310-314.

26. Jones NL, Roifman CM, Griffiths AM, Sherman P. Ketotifen therapy for acute ulcerative colitis in children: A pilot study. Dig Dis Sci 1998; 43: 609-615.

27. Schwartz DA, Pardi DS, Murray JA. Use of montelukast as esteroidsparing agent for recurrent eosinophilic gastroenteritis. Dig Dis Sci 2001; 46: 1787-1790.

28. Bastida Eizaguirre M, Iturbe Ortiz de Urbina R, Arto Urzainqui MJ, de las Muñecas Ibarrechebea J, Beltrán de Heredia P. Gastroenteritis eosinofílica en un paciente afectado de osteodistrofia hereditaria de Albright. An Esp Pediatr 2002; 56: 576-578.

29. Cózar Ibáñez A, Olmo Escribano M, Bernet Vegue E, González Olid MD, Gómez Ortega. Gastroenteritis eosinofílica y cirugía. Cir Esp 1996; 60: 41-44. 